\title{
Authors on the rise
}

\author{
We look at changes in authorship and cross-institutional links in the papers we publish. Both are increasing as the \\ geosciences continue to become more collaborative.
}

G eoscience spans disciplines, and so do our authors. Authors assembled from different fields and institutions show the value of collaboration, though authorship is, as ever, also affected by the complex, evolving norms of the research community. In this Editorial, we explore how and why author lists have grown in Nature Geoscience over time.

There is no simple authorship formula for Nature Geoscience papers. We expect each author to have substantially contributed to a study, in line with our guidelines. Otherwise, we leave it up to our authors to build the team needed to develop and tackle cutting-edge research questions.

The geosciences are getting more multidisciplinary a factor that might be expected to influence the authorship of the studies we publish. We examined our archives to see how author lists have changed since the start of Nature Geoscience, and compiled the total number of authors and affiliations for papers published every other year from 2008 to 2020 . For simplicity, all affiliations were counted, including those of individual authors associated with multiple institutions or departments at the same university.

There has been a striking increase in the mean number of authors per paper in recent years (Fig. 1). This is in part due to a small number of papers with large numbers of authors. However, a smaller increase in the median number of authors per paper - from five in 2008 to seven in 2020 suggests that these outliers aren't the only cause of this upward trend.

Mean affiliations per paper have similarly gone up. This isn't unexpected given that the number of authors and affiliations are strongly, but not entirely, correlated. However, this shows that papers in our journal increasingly have authors from a greater mix of departments and institutions.

The cause of these trends is difficult to pinpoint, but myriad factors could be playing a role. We know that international collaborations have become more frequent in papers we publish. There's also been a drive to better integrate formerly siloed fields exemplified by the rise of the Earth System Science concept, while more of our papers are arising from large, interdisciplinary projects such as planetary science missions. Furthermore, the range

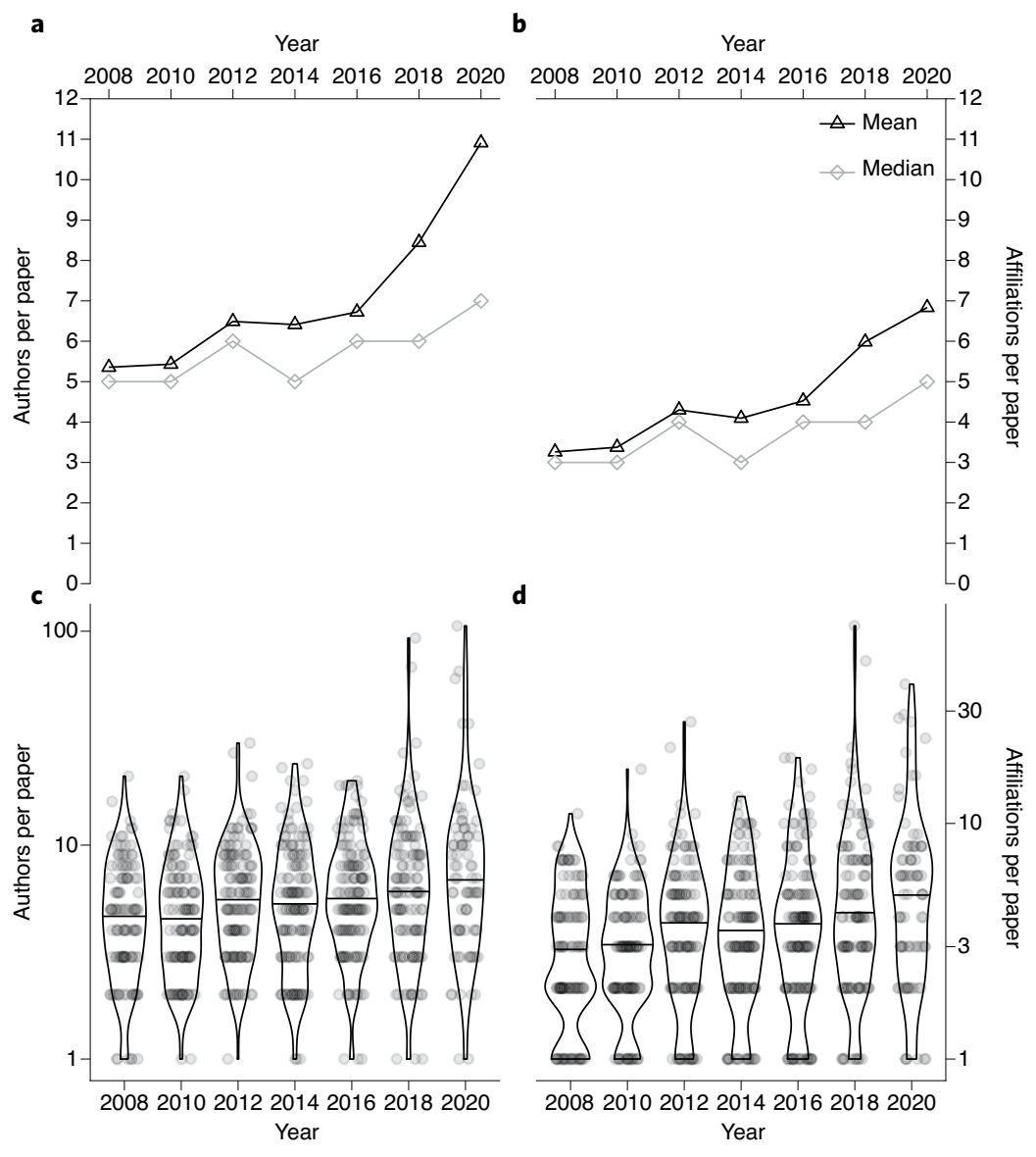

Fig. 1 | Authors and affiliations in Nature Geoscience papers for every other year since 2008. a,b, The mean and median number of authors (a) and affiliations (b) for papers in a given year. $\mathbf{c}, \mathbf{d}$, The grey circles show the total number of authors (c) and affiliations (d) data for each paper binned by year. Note these are plotted on a log scale. The distribution of the data is represented by vertical black lines, while horizontal lines represent the mean. Number of affiliations can include separate departments at the same university as well as multiple affiliations associated with individual authors.

of methodologies in individual studies has grown in ways that might explain the need for author lists covering a wider range of technical expertise.

Our data do not represent the geoscience literature as a whole. This means that our own editorial expectations, and those of our reviewers, of what a study needs to achieve to be suitable for Nature Geoscience may have changed over time, both due to the evolution of the science itself and increasing selectivity as our submissions have grown.

This analysis is not meant to discourage submission of papers with a small number of authors. In this year alone, our published papers have ranged from 1 to over 100 authors. Important, well-supported findings will always have a place in our journal.

Having more authors with more affiliations can present practical difficulties in bringing a study to publication. Still, untangling many of the big, open questions in the Earth and planetary sciences will probably require increased levels of collaboration - across disciplines, skill sets, countries, and diverse viewpoints.

Published online: 1 September 2020 https://doi.org/10.1038/s41561-020-0632-z 